\title{
Deep Learning-Based Automatic Segmentation of Lumbosacral Nerves on CT for Spinal Intervention: A Translational Study
}

\author{
(D) G. Fan, (D) H. Liu, (D) Z. Wu, (D) Y. Li, (D) C. Feng, DD. Wang, (D). Luo, DW.M. Wells III, and DS. He
} O- $=$

\begin{abstract}
BACKGROUND AND PURPOSE: 3D reconstruction of a targeted area ("safe" triangle and Kambin triangle) may benefit the viability assessment of transforaminal epidural steroid injection, especially at the L5/S1 level. However, manual segmentation of lumbosacral nerves for 3D reconstruction is time-consuming. The aim of this study was to investigate the feasibility of deep learning-based segmentation of lumbosacral nerves on $\mathrm{CT}$ and the reconstruction of the safe triangle and Kambin triangle.
\end{abstract}

MATERIALS AND METHODS: A total of 50 cases of spinal CT were manually labeled for lumbosacral nerves and bones using Slicer 4.8 . The ratio of training/validation/testing was 32:8:10. A 3D U-Net was adopted to build the model SPINECT for automatic segmentations of lumbosacral structures. The Dice score, pixel accuracy, and Intersection over Union were computed to assess the segmentation performance of SPINECT. The areas of Kambin and safe triangles were measured to validate the 3D reconstruction.

RESULTS: The results revealed successful segmentation of lumbosacral bone and nerve on CT. The average pixel accuracy for bone was 0.940 , and for nerve, 0.918 . The average Intersection over Union for bone was 0.897 and for nerve, 0.827 . The Dice score for bone was 0.945 , and for nerve, it was 0.905 . There were no significant differences in the quantified Kambin triangle or safe triangle between manually segmented images and automatically segmented images $(P>.05)$.

CONCLUSIONS: Deep learning-based automatic segmentation of lumbosacral structures (nerves and bone) on routine CT is feasible, and SPINECT-based 3D reconstruction of safe and Kambin triangles is also validated.

ABBREVIATIONS: ESI = epidural steroid injection; loU = Intersection over Union; tESI = transforaminal epidural steroid injection

ow back pain with a radicular component is a common ailment in many clinics and is a leading cause of disability and adds a great burden to health care costs globally. ${ }^{1,2}$ It is difficult to

Received January 14, 2019; accepted after revision April 16.

From the Orthopedic Department, Shanghai Tenth People's Hospital (G.F., C.F., D.W., S.H.), Tongji University School of Medicine, Shanghai, China; Department of Spine Surgery (G.F.), Third Affiliated Hospital of Sun Yatsen University, Guangzhou, China; Surgical Planning Lab (G.F., J.L., W.M.W.), Radiology Department, Brigham and Women's Hospital, Harvard Medical School, Boston, Massachusetts; Spinal Pain Research Institute of Tongji University (H.L., C.F., D.W., S.H.), Shanghai, China; School of Data and Computer Science (Z.W.), Sun Yat-sen University, Guangzhou, China; Shanghai Jiao Tong University School of Medicine (Y.L.), Shanghai, China; and Graduate School of Frontier Sciences (J.L.), University of Tokyo, Tokyo, Japan. G. Fan and H. Liu contributed equally to the study.

This work is supported by the China Scholarship Council (201706260169), Shanghai Hospital Development Center (16CR3017A), and National Institutes of Health grant P41EB015898 (W.M.W.).

Please address correspondence to Shisheng He, MD, and Guoxin Fan, MD, 301 Yanchang Rd, Shanghai 200072, China; e-mail: tjhss7418@tongji.edu.cn, gfan@tongji.edu.cn

-- Indicates open access to non-subscribers at www.ajnr.org

三 Indicates article with supplemental on-line appendix and table.

http://dx.doi.org/10.3174/ajnr.A6070 detect the specific etiology, but the pain is usually caused by inflammation of nerves due to mechanical compression or chemical irritation. ${ }^{3}$ Lumbar degenerative disease, including spinal stenosis and lumbar disc herniation, is a main culprit of low back pain and radicular symptoms. Before spinal surgery, epidural steroid injection (ESI) is an interventional option for the management of low back and radicular pain. ${ }^{4,5}$ Transforaminal epidural steroid injection (tESI) is a well-validated technique with merits of large distributions of injectate and superior short-term pain relief compared with an interlaminar or caudal approach. ${ }^{6,7}$ Typically, tESI is conducted via a "safe" triangle, while others prefer via a Kambin triangle because the safe triangle has been found to sometimes not be safe. ${ }^{8}$ The Kambin triangle is defined as an anatomic triangle with the nerve root (hypotenuse), traversing nerve root and/or facet joint (height), and superior border of a lower vertebra (base) as its components. ${ }^{9}$ The safe triangle is defined as the lower margin of the pedicle (base), the lateral border of the vertebral body (height), and the outer border of the exiting nerve (hypotenuse). ${ }^{10}$ Because the 2 triangles are $3 \mathrm{D}$, the targeted area of $\mathrm{tESI}$ is variable from different views or even totally concealed by bony 


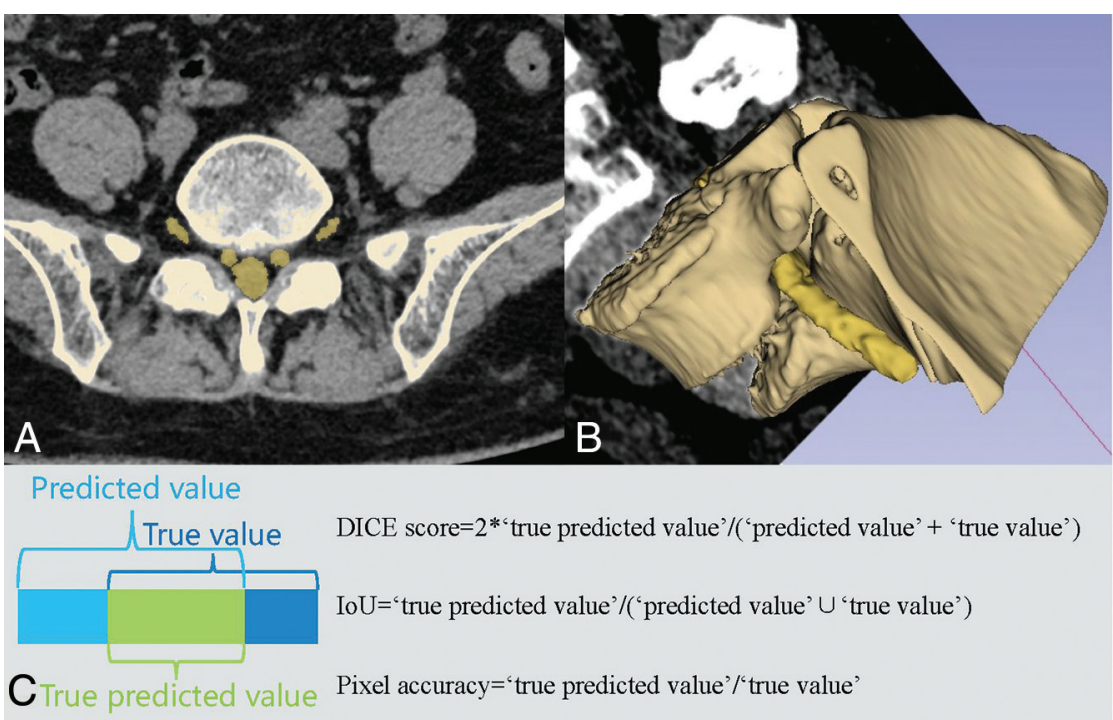

FIG 1. Manual segmentation and 3D reconstruction on Slicer. $A$, manual labels. $B, 3 D$ reconstruction with a coronal image. $C$, Illustrations of the Dice score, Intersection-over-Union, and pixel accuracy.

structures such as a high iliac crest, enlarged transverse process, and facet joint, especially at the L5/S1 level. ${ }^{10-12}$ An increased area of the safe triangle and Kambin triangle should lead to more frequent successful outcomes of tESI. ${ }^{10}$ Complications of tESI such as intravascular injection and nerve root injury cause serious problems. $^{13,14}$

$\mathrm{X}$-ray fluoroscopy is the criterion standard to guide tESI. ${ }^{15}$ The bony landmark "Scotty dog" is identified to estimate the targeted area to present larger areas of the safe triangle or Kambin triangle. The $\mathrm{C}$-arm machine is usually tilted to a certain angle to conduct an oblique fluoroscopy to identify the Scotty dog. ${ }^{16}$ However, $\mathrm{x}$-ray fluoroscopy is incapable of providing information about soft tissues and localizing the needle tip in a real-time manner. Thus, CT fluoroscopy is introduced to guide the tESI because it can quickly and safely localize needles and minimize the risk of nerve injury. ${ }^{17}$ Usually, an initial CT scan with good image quality is obtained for viability assessment of the tESI, during which an ideal needle trajectory is planned on a single axial CT slice. ${ }^{18}$ Then, a CT fluoroscopy technique is used to guide the needle advancement on a single CT slice during which soft tissue is poorly presented on images. ${ }^{18}$ Therefore, a larger targeted area of needle advancement should reduce the risk of injuring the neurovascular structure. However, the CT plane is incapable of directly and quickly identifying an accessible trajectory targeting the largest safe or Kambin triangle, and sometimes the CT plane presents no accessible pathway on all axial slices. Instead, 3D construction of the safe or Kambin triangle may benefit the viability assessment of the tESI. Free manipulation of the 3D model will quickly identify an oblique needle pathway targeting the large safe triangle or Kambin triangle. However, manual segmentation of lumbosacral nerves for $3 \mathrm{D}$ reconstruction is time-consuming.

Recently, deep learning has gained substantial attention in the field of radiology. ${ }^{19-22}$ Deep learning algorithms can learn from large amounts of data using neural networks, frequently convolutional neural networks. ${ }^{23} \mathrm{Al}-$ though convolutional neural networks were proposed decades ago, it is only in the past 7 years that deep learning has achieved great success due to massive available data, increased processing power, and rapid development of algorithms. ${ }^{23}$ The U-Net (https:// lmb.informatik.uni-freiburg.de/peo ple/ronneber/u-net/) is a kind of convolutional neural network that was developed for biomedical image segmentation by Ronneberger et $\mathrm{al}^{24}$ in 2015, and it has many applications in the segmentation of $2 \mathrm{D}$ images. In 2016, the same group developed a 3D U-Net (https://www.researchgate.net/ publication/304226155_3D_U-Net_ Learning_Dense_Volumetric_Segmenta tion_from_Sparse_Annotation) for volumetric segmentation as an extension architecture of the U-Net. ${ }^{25}$ Many studies have validated the segmentation performance of the $3 \mathrm{D}$ U-Net for volumetric medical images. ${ }^{26-28}$ To the best of our knowledge, no studies are available on achieving automatic segmentation of lumbosacral nerves on CT. The aim of this study was to investigate the feasibility of automatically segmenting lumbosacral structures (nerves and bone) on CT with the $3 \mathrm{D}$ U-Net and the reconstruction of the safe and Kambin triangles.

\section{MATERIALS AND METHODS}

This retrospective study was Health Insurance Portability and Accountability Act-compliant and approved by the institutional ethical committee of Shanghai Tenth People's Hospital before data extraction. A total of 50 patients with thin-layer CT covering the lumbosacral level from Shanghai Tenth People's Hospital were included in this study. All algorithms were developed and tested using Keras (Version 2.1.1 with tensorflow_backend.py; http://pydoc.net/Keras/ 2.1.2/keras.preprocessing.image/) in a personal computer (graphics processing unit: a Nvidia GeForce 1080Ti, https://www.nvidia.com/ en-us/geforce/products/10series/geforce-gtx-1080-ti/, with 4 GB of memory and a 3.5-GHz Intel Core i7-4790 CPU with 8 GB of memory).

\section{Manual Annotations}

All data were manually segmented with Slicer 4.8 (http://www. slicer.org). ${ }^{29}$ Lumbosacral nerves and bones were meticulously segmented and labeled (Fig $1 A,-B$ ). A spine surgeon who is an expert in reading lumbar CT and had systematic training in Slicer manually segmented the nerves and bones. Then the segmented images were reviewed by an interventional radiologist and pain physician, both of whom are experts in CT-guided epidural steroid injection. Any disagreements of segmentation were solved by the 3 doctors voting. These manual annotations were regarded as the ground truth. 


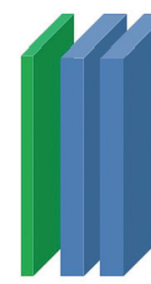

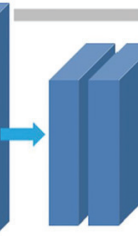

6464

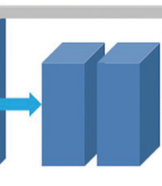

$128 \quad 128$

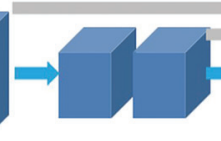

$256 \quad 256$

512

$\begin{array}{lll}1 & 32 & 32\end{array}$

Input $32 \times 64 \times 64 \times 1$
Output $32 \times 64 \times 64 \times 3$
$3 \mathrm{D} \operatorname{conv}(3 \times 3 \times 3)+\mathrm{bn}+\mathrm{relu}$

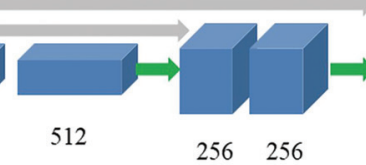

512$$
256
$$

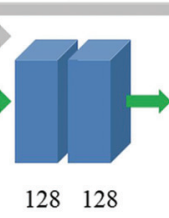

6464

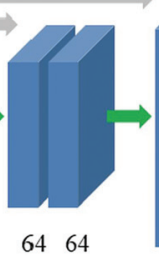

3232

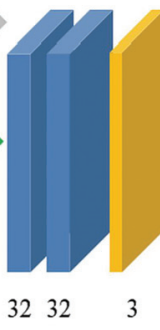

FIG 2. Schematic of the network architecture.

\section{Table 1: Overview of the combined algorithm}

Algorithm 1 : Combined Algorithm
Require: $X: C T$ volume, shape $=\mathrm{D} \times \mathrm{H} \times \mathrm{W}$
Require: $x_{i}=X\left(L_{i}\right), \quad(i=1, \ldots, k): C T$ voxel patch
Require: $\mathbf{y}_{i}=M\left(x_{i}\right): y_{i}$ is the output of the last layer (softmax
activation function) of the model $M, y_{i}$ has 1 more dimension
than $x_{i}$, and this dimension has 3 channels. Each channel refers
to the probability of the corresponding voxel belonging to
background or bone or nerve, respectively.
1) Initialize: $\mathbf{Y} \leftarrow \mathbf{0}$
2) For $\mathbf{x}_{i} \in X,(i=1, \ldots, k)$ do
3) $Y\left(L_{i}:\right)+=y_{i}$
4) End for
5) $S \leftarrow$ arg max $(Y$, axis $=-1)$ (find the channel with the largest
value in the last dimension)
6) Return $S$ (the automatic mask)

Note:- $M$ indicates the model (network); L, location of the CT voxel patch $x$ at the CT volumn $X ; Y$, summed probability; max, maximum.

\section{Data Preprocessing and Segmentation Assessment}

All thin-layer CT scans were preprocessed using the following steps: resampling, cropping, and intensity normalization (Online Appendix). Pixel accuracy, Intersection over Union (IoU), and the Dice score were used to assess the segmentation performance of lumbosacral structures (Fig 1C). The functions of these 3 indicators are as follows:

Dice Score $=2 \times$ True Predicted Voxels/(Predicted Voxels + True Voxels)

IoU $=$ True Predicted Voxels/(Predicted Voxels $\cup$ True Voxels)

Pixel Accuracy $=$ True Predicted Voxels/True Voxels.

Because the Dice score is the most common indicator in computer vision, ${ }^{30}$ the current study adopted it as the primary index to assess the segmentation performance.

\section{Network Architecture}

The 3D U-Net was adopted for multiclass segmentation of lumbosacral structures (Fig 2). The adopted network consists of 2 parts, the encoder and the decoder parts. The encoder part performs data analysis and feature-representation learning from the input data, and the decoder part generates segmentation results. There are also 4 shortcut connections (concatenations) between layers of equal resolution in the encoder and decoder paths. The last layer of the model is a $1 \times 1 \times 1$ convolutional layer followed by a softmax layer (https://www.mathworks.com/help/deeplearning/ref/nnet.cnn. layer.softmaxlayer.html), with 3 output channels. The input of the model is $32 \times 64 \times 64$ voxel patches of CT. The output is the corre- sponding probability mask, and its shape is $32 \times 64 \times 64 \times 3$. The whole architecture has 22,581,411 parameters. The developed model is named SPINECT because it aims to automatically segment multiple structures solely on the basis of spinal CT.

\section{Training}

During the training phase, a number of minibatch CT and manual labeling masks are randomly selected from the training dataset, and the image data are subjected to the standardization processing and the augmentation operation. As a result, the training data input to each training iteration is different, and this feature improves the generalization ability of the model. The convolutional layer parameters of 3D U-Net are initialized by the method of $\mathrm{He}$ et al. ${ }^{31}$ The size of the patch (depth $\times$ height $\times$ width) input to the $3 \mathrm{D}$ U-Net during training is $32 \times 64 \times 64$ (unit:voxel); the minibatch is 4 , which is optimized by the Adam optimization algorithm $^{32}$; and the learning rate is $5 \mathrm{e}-4$. We selected the Adam optimization algorithm instead of stochastic gradient descent for training the deep learning network because it was straightforward to implement and computationally efficient.

\section{Five-Fold Validation}

During model training, 1 validation was performed every 100 training iterations. Specifically, 6 cases were randomly selected from the validation dataset for standard processing. The standard processing included several sequential steps: 1) Set the voxel size to $1 \mathrm{~mm}$ by the nearest interpolation method, and 2) standardization. Then, we added random noise plus random horizontal and vertical flipping to augment the selected cases. A sliding window of $32 \times 64 \times 64$ was used to traverse the data with stride $=(20 \times 40 \times 40)$ to obtain the patch. The patch is input to the current model; then the model generates the corresponding probability mask. Finally, the automatic segmented mask is obtained with a combined algorithm (Table 1). The Dice score of each voxel class is obtained with comparison of the automatic segmented mask and the manually labeled mask. If the average of the Dice score is greater than the prior best Dice score by the current iteration, the current model parameters will be saved. We use a combined algorithm in which the patch-based CNN is applied at a sequence of patches of the data, and the resulting label probabilities are summed according to their voxel location. Subsequently, the labels are established by the probabilities. 


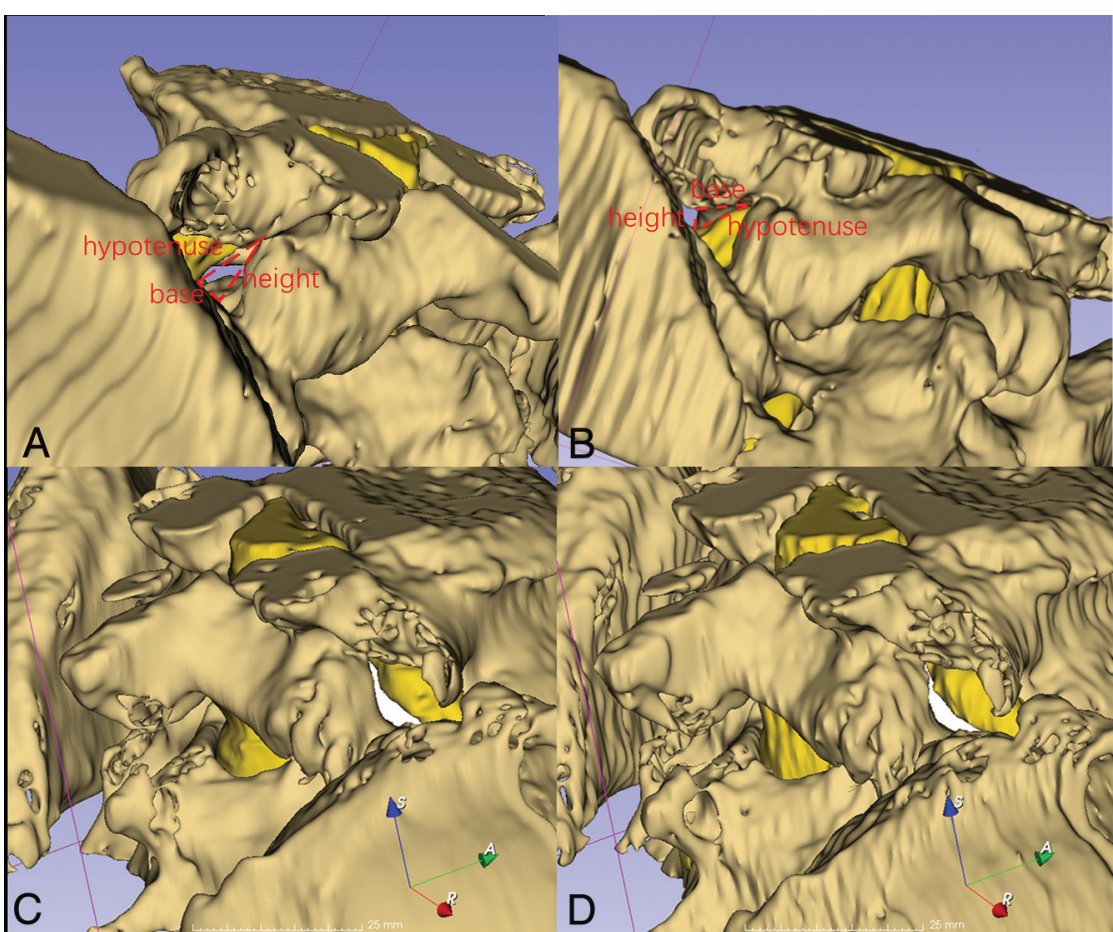

FIG 3. Measurement of safe and Kambin triangles. $A$, Schematics of the Kambin triangle. $B$, Schematics of the safe triangle. $C$, Measurement of the Kambin triangle on a manually segmented image. $D$, Measurement of the safe triangle on automatically segmented images.

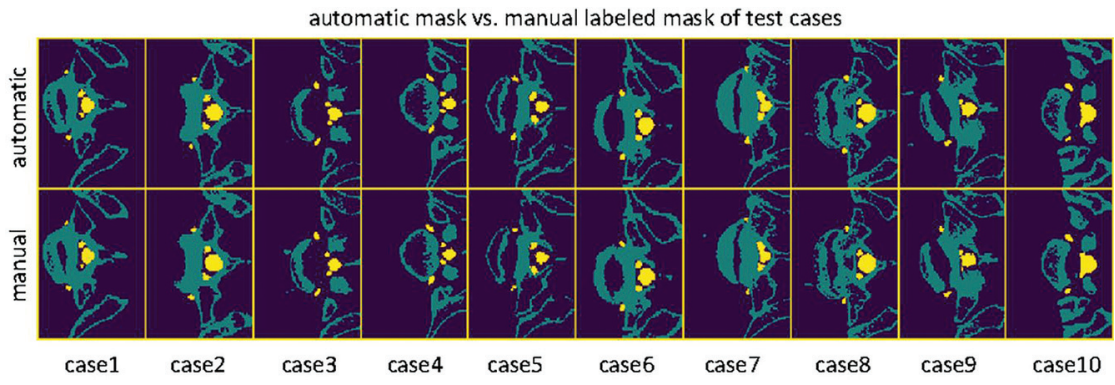

FIG 4. Automatic and manually labeled masks.

Table 2: Segmentation accuracy in 10 testing cases $^{\mathrm{a}}$

\begin{tabular}{lccc}
\hline Structures & Pixel Accuracy (\%) & IoU (\%) & Dice Score (\%) \\
\hline Bones & $94.05 \pm 6.68$ & $89.73 \pm 4.32$ & $94.54 \pm 2.43$ \\
& $(82.0-99.9)$ & $(82.0-95.2)$ & $(90.1-97.5)$ \\
Nerves & $91.43 \pm 3.48$ & $82.71 \pm 3.25$ & $90.51 \pm 1.94$ \\
& $(85.3-94.4)$ & $(76.3-87.4)$ & $(86.6-93.2)$ \\
\hline
\end{tabular}

${ }^{a}$ Data are means and percentages unless otherwise noted.

\section{Testing}

The ratio of training, validation, and testing of the 50 cases from our local dataset is 32:8:10. A total of 10 cases randomly selected from the local dataset were tested on SPINECT. To validate the robustness of our trained model, we also conducted cross-dataset testing on 10 cases from an on-line imaging dataset (SpineWeb; http://spineweb.digitalimaginggroup.ca/). ${ }^{33}$ Pixel accuracy, IoU, and the Dice score were used to assess the segmentation performance of lumbosacral structures.

\section{Radiographic Evaluation}

To compare the difference between manually segmented masks and automatically generated masks, we present a random slice from each case. In addition, 3D rendering of manual masks before preprocessing, post-preprocessing masks, automatically generated masks, as well as smoothed 3D rendering of automatically generated masks (smoothing method: median; Kernel size: $1.5 \mathrm{~mm}$ ) are also presented. To further investigate the segmentation performance of SPINECT, we measured both Kambin and safe triangles at the L5/S1 level on both sides on manually segmented and automatically segmented images (Fig 3). First, the Kambin triangle and safe triangle were visualized on Slicer with 3D rendering of manually and automatically segmented labels. Then, the Kambin triangle and safe triangle were set with the same angles between manually and automatically segmented images. Screenshots were obtained and imported into Image J software (Version 1.8.0_112; National Institutes of Health, Bethesda, Maryland) for area measurements of the Kambin and safe triangles. Two independent observers measured the area of the 2 triangles from manually and automatically segmented images. One month later, one of the observers measured the area of the 2 triangles again. The intraclass correlation coefficient was calculated to assess test-retest reliability and interobserver reliability of multiple measurements.

\section{Statistical Analysis}

The generated labels from SPINECT were used to generate the $3 \mathrm{D}$ rendering of bones and nerves on Slicer. After testing the normal distribution (Shapiro-Wilk test), we used a paired Student $t$ test to detect statistically significant differences of the safe and Kambin triangles between manually segmented images and automatically segmented images. All continuous data are presented as mean $\pm \mathrm{SD}$, and a $P$ value $<.05$ is a statistically significant difference.

\section{RESULTS}

Testing results revealed that SPINECT could achieve successful segmentation of multiple structures (bones and nerves) on CT (Fig 4). Quantitative segmentation accuracy is shown in Table 2. The average pixel accuracy for the bones was 0.940 , and for the nerves, 0.918 . The average IoU for the bones was 0.897 , and for the nerves, it was 0.827 . The Dice score for the bones was 0.945 , and for the nerves, it was 0.905 . In each validation fold, it took about 4 hours 35 minutes to finish the training of the segmentation net. After training, SPINECT requires a total RAM space of 305.384 $\mathrm{MB}$, and it takes about 3.1 seconds (Nvidia GeForce 1080Ti) to complete an automatic segmentation on a single case (On-line 
Table 3: Segmentation accuracy in 10 testing cases from the open dataset $^{\mathrm{a}}$

\begin{tabular}{lccc}
\hline Structures & Pixel Accuracy (\%) & IoU (\%) & Dice Score (\%) \\
\hline Bones & $99.62 \pm 0.35$ & $81.40 \pm 11.33$ & $89.34 \pm 7.28$ \\
& $(99.3-99.9)$ & $(60.5-93.3)$ & $(75.42-96.5)$ \\
Nerves & $87.74 \pm 4.82$ & $80.64 \pm 3.31$ & $89.25 \pm 2.00$ \\
& $(79.4-93.1)$ & $(75.5-82.9)$ & $(88.1-93.4)$ \\
\hline
\end{tabular}

${ }^{a}$ Data are means and percentages unless otherwise noted.

Table 4: Measured area of the safe and Kambin triangles ${ }^{a}$

\begin{tabular}{cccc}
\hline $\begin{array}{c}\text { Area } \\
\left(\mathbf{m m}^{2}\right)\end{array}$ & Manual Images & Automatic Images & $\begin{array}{c}\boldsymbol{P} \\
\text { Value }\end{array}$ \\
\hline $\begin{array}{c}\text { Kambin } \\
\text { triangle }\end{array}$ & $37.80 \pm 20.90(15.11-87.51)$ & $36.41 \pm 19.27(11.46-78.63)$ & .302 \\
$\begin{array}{c}\text { Safe } \\
\text { triangle }\end{array}$ & $8.69 \pm 2.24(6.04-13.27)$ & $8.56 \pm 3.25(3.18-17.91)$ & .792 \\
\hline
\end{tabular}

${ }^{a}$ Data are means and percentages unless otherwise noted.

\begin{tabular}{|c|c|c|}
\hline $\begin{array}{c}\text { Intraclass Correlation } \\
\text { Coefficient }\end{array}$ & $\begin{array}{l}\text { Test-Retest } \\
\text { Reliability }\end{array}$ & $\begin{array}{c}\text { Interobserver } \\
\text { Reliability }\end{array}$ \\
\hline \multicolumn{3}{|c|}{$3 \mathrm{D}$ rendering of manual segmentation } \\
\hline Kambin triangle & 0.983 & 0.984 \\
\hline Safe triangle & 0.881 & 0.922 \\
\hline \multicolumn{3}{|c|}{$3 \mathrm{D}$ rendering of automatic segmentation } \\
\hline Kambin triangle & 0.988 & 0.982 \\
\hline Safe triangle & 0.977 & 0.959 \\
\hline
\end{tabular}

Table). In total, it took about 13 seconds to process 1 set of CT data (from data uploading and data preprocessing to semantic segmentation) with a graphics processing unit (Nvidia GeForce $1080 \mathrm{Ti}$ ), which is far less than the 30 minutes for manual segmentation. Besides, the segmentation performance of 10 cases from SpineWeb was similar to that of our testing dataset (Table 3).

More important, there were no significant differences in the measured area of the safe or Kambin triangle between manual images and automatic images (Table 4). The area of Kambin triangle was $37.80 \pm 20.90 \mathrm{~mm}^{2}$ on manual images and $36.41 \pm$ $19.27 \mathrm{~mm}^{2}$ on automatic images $(P=.302)$. The area of the safe triangle was $8.69 \pm 2.24 \mathrm{~mm}^{2}$ on manual images and $8.56 \pm 3.25$ $\mathrm{mm}^{2}$ on automatic images $(P=.792)$. The reliability test revealed strong test-retest reliability and interobserver reliability of multiple measurements (Table 5). As in Fig 5, 3D rendering of automatic masks was similar to that of post-preprocess masks, and 3D rendering of manual masks was similar to that of smoothed automatic masks. Moreover, dura compression was clearly present in the four $3 \mathrm{D}$ rendered images.

\section{DISCUSSION}

Knowledge of bony and neurovascular anatomy of the spinal foramen is essential when performing tESI. ${ }^{34}$ However, the Kambin or safe triangle has never been visualized before tESI. Thin-layer CT has great potential for reconstructing the safe and Kambin triangles, whereas manual segmentation of nerve roots is timeconsuming. The current study has validated the feasibility of automatically segmenting lumbosacral structures (nerves and bone) on CT through deep learning and the $3 \mathrm{D}$ reconstruction of Kambin and safe triangles. To the best of our knowledge, this is the first study automatically segmenting lumbosacral nerves on CT through deep learning.

Reconstruction of the safe or Kambin triangle may benefit the

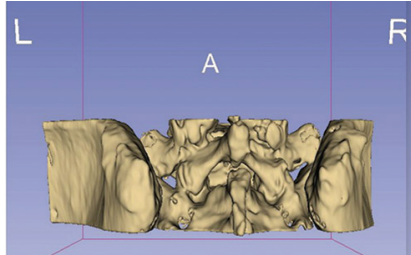

FL
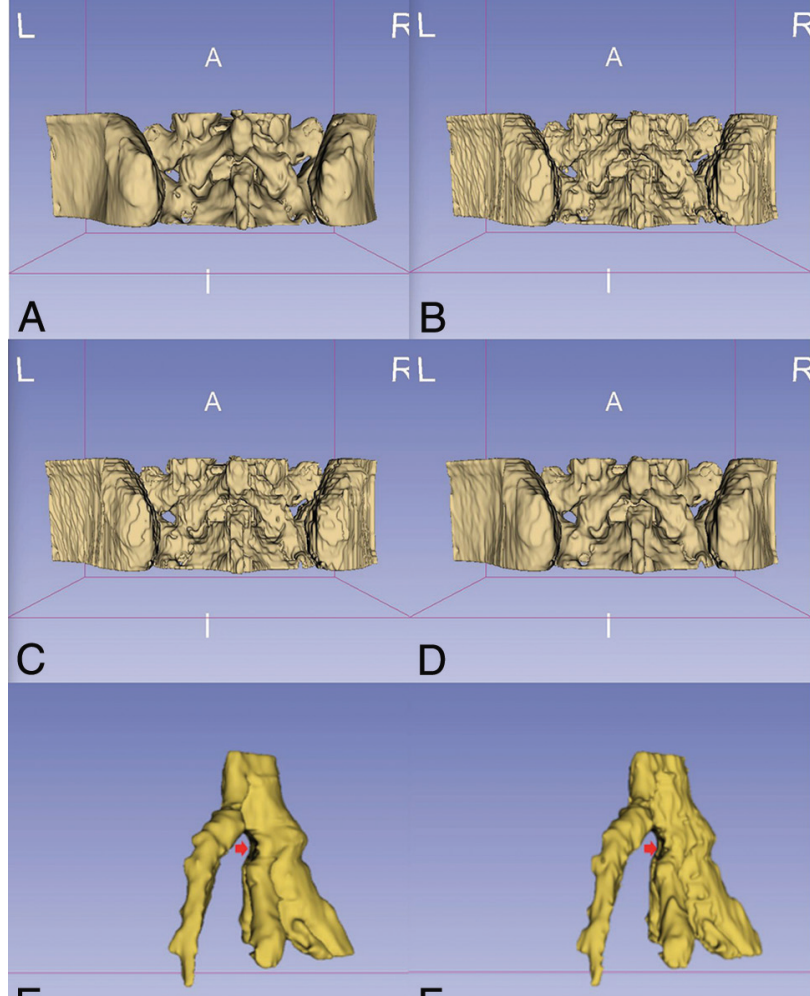

E
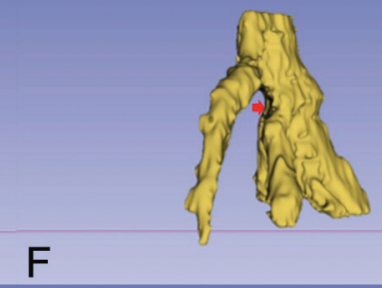

$\mathrm{F}$

G

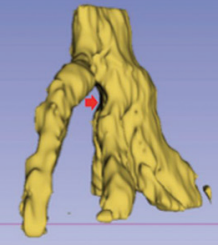

$\mathrm{H}$

FIG 5. 3D rendering of automatic masks and manually labeled masks of bones and nerves. A, 3D rendering of manual masks before preprocessing. $B, 3 D$ rendering of post-preprocessed masks. $C, 3 D$ rendering of automatically generated masks. $D$, Smoothed $3 D$ rendering of automatically generated masks. $E, 3 D$ rendering of manual masks before preprocessing (arrow indicates the compressed dura). $F, 3 D$ rendering of post-preprocessed masks (arrow indicates the compressed dura). $G, 3 D$ rendering of automatically generated masks (arrow indicates the compressed dura). $\mathrm{H}$, Smoothed 3D rendering of automatically generated masks (arrow indicates the compressed dura).

viability assessment of tESI, especially at the L5/S1 level. Ideally, the needle trajectory is planned on an axial plane of conventional CT slices. ${ }^{10}$ However, trajectory planning could be difficult at the L5/S1 level in some cases due to the bony obstacles such as the iliac crest, facet joint, and transverse process (Fig 6). In that case, an accessible pathway could be found in an oblique perspective. However, operators may fail to quickly identify an oblique trajectory targeting the largest safe or Kambin triangle, even if the CT gantry can be tentatively angled caudally or cranially. Another option is to change the targeted area (eg, from the safe to the Kambin triangle) for trajectory planning. ${ }^{35}$ Nevertheless, axial CT slices are still incapable of presenting a large safe or Kambin triangle directly and quickly. Thus, 3D reconstruction of the safe or Kambin triangle may facilitate the viability assessment of tESI because it can quickly provide the specific perspective with a large 


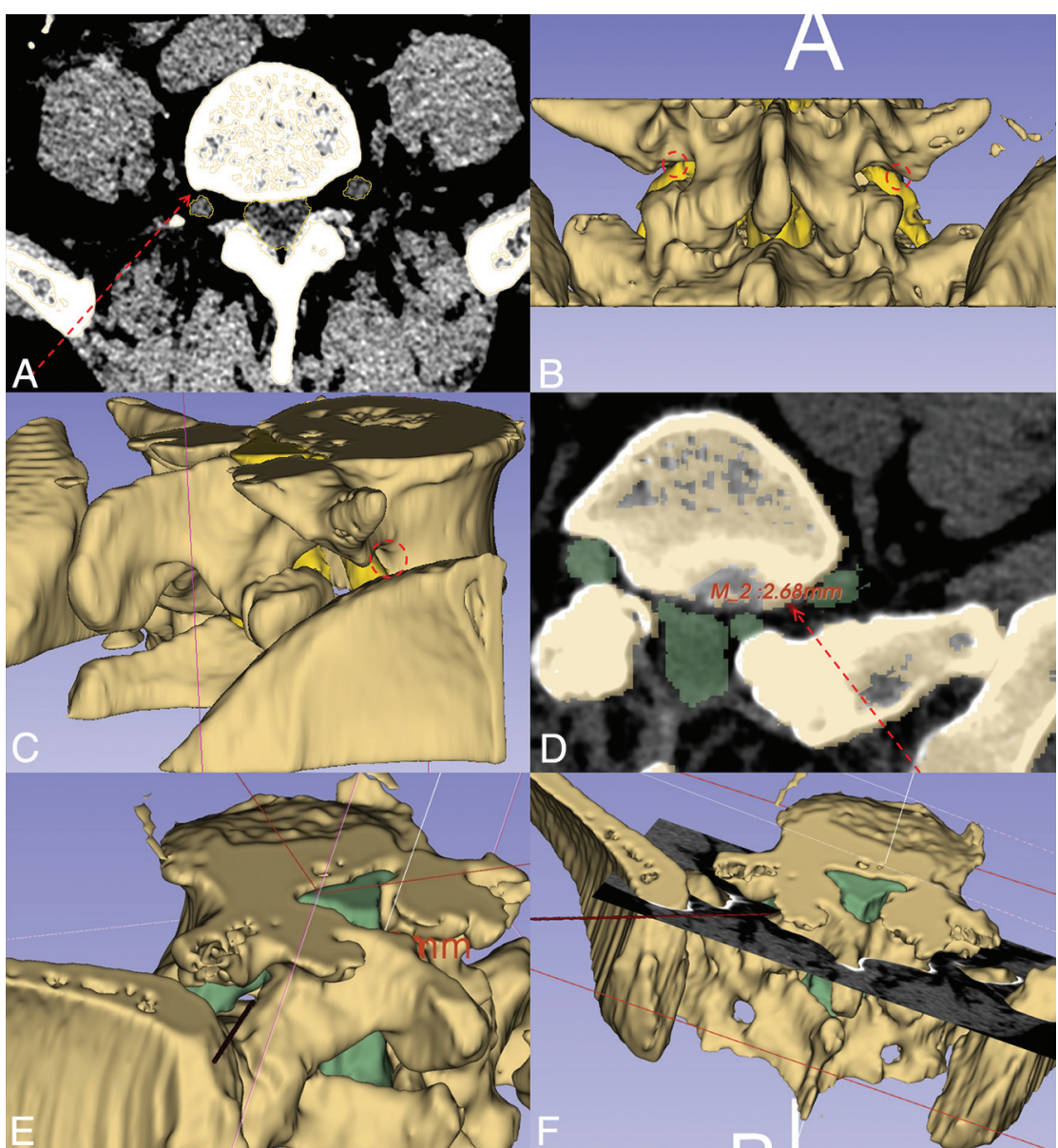

FIG 6. 3D model-based viability assessment of a transforaminal epidural steroid injection. $A$, Inaccessible trajectory to the safe triangle on an axial CT slice. $B$, Minimal space of the safe triangle on the posterior 3D model. C, Accessible oblique trajectory to the safe triangle on the 3D model. $D$, Inaccessible trajectory to the Kambin triangle on axial CT slice. E, Accessible trajectory on the 3D model. F, Oblique trajectory-guided nonaxial CT plane.

targeted area. Ra and $\mathrm{Min}^{10}$ tried to identify an optimal angle of a nonaxial needle path with the largest area of the safe triangle for $\mathrm{x}$-ray fluoroscopic guidance, but others thought a specific needle path is dictated by specific patient anatomy and cannot be consistently predicted using a constant angle. With free manipulation of 3D-rendering models, an oblique needle path could be planned when a large area of the safe triangle or Kambin triangle is presented (Fig 6C, -E). Our study also quantified the area of the safe or Kambin triangle, which might also help assess the viability of specific approaches. The area of the safe triangle ranged from 4 to $18 \mathrm{~mm}^{2}$ in our series, which is similar to that in the previous study. ${ }^{10}$ The area of the Kambin triangle at the L5/S1 level ranged from 10 to $84 \mathrm{~mm}^{2}$ in our series, which is smaller than the results from other studies ${ }^{36,37}$ because others removed the facet joint for measurement. More important, the measurements of triangles in our study confirmed no significant differences between manually segmented images and automatically segmented images, which validates the $3 \mathrm{D}$ reconstruction based on SPINECT in medical indicators.

Nerve segmentation is essential for visualizing the safe and Kambin triangles because the exiting nerve root is the hypotenuse of the 2 triangles. Physicians usually recognize the Scotty dog to estimate the location of the safe and Kambin triangles under the guidance of $\mathrm{x}$-ray fluoroscopy because the exiting nerve root has not yet been outlined. Moreover, only after multiple attempted punctures will contrast be injected to show the epidural and perineural flow, which may finally outline the nerve root sheath and epidural flow. Thus, $\mathrm{x}$-ray fluoroscopy fails to demonstrate the detailed location of the exiting nerve root before punctures. The main advantage of CT is that it is good at precisely locating the needle placement using 3D imaging of soft tissues and osseous anatomy. ${ }^{38}$ Moreover, CT fluoroscopy is also capable of demonstrating real-time contrast flow. Nevertheless, nerve segmentation is not yet available on the current CT workstations for ESI. Diffusion tensor imaging or the MR neurography technique may be useful to enhance the spinal nerves for diagnosis and surgical planning. ${ }^{37,39}$ However, DTI or MR neurography scanning can be lengthy and costly and is not practical for ESI. In this current study, however, we have achieved automatic and precise segmentation of lumbosacral nerves on routine CT and successfully visualized the safe and Kambin triangles with segmented bones and nerves.

As more medical imaging datasets are created by medical experts, the application of deep learning in radiology is growing because of its excellent performance in recognition and segmentation. While bony structures are easily identified in intraoperative practice, deep learning may enable rapid recognition and precise segmentation of important soft tissue. Moreover, all paraspinal structures (eg, nerves, vessels, discs, muscles, ligaments) may also play an important role in procedure planning, spinal navigation, and even robotic surgery in the near future. The current study has shown that thin-layer CT is a good candidate for segmenting nerves, which discloses the substantial potential of CT in segmenting spinal soft tissue. Moreover, the performance of SPINECT further supports the findings of other similar studies using U-Net and its variants for semantic segmentation of biomedical images. ${ }^{40,41}$ We adopted the 3D UNet instead of the 2D U-Net because 3D U-Net can adopt the information between slices of the CT volume. However, the UNet has limitations of class imbalance and decreased segmentation accuracy. Because the voxels of background, bone, and nerve are quite different, small structure (nerve) tends to have a lower Dice score compared with large structures (bone and background) in semantic segmentation. Thus, we adopted the weighted softmax cross-entropy loss function, and the outcomes seemed to be acceptable. Feng et $\mathrm{a}^{42}$ used the U-Net to segment the spinal cord on thoracic CT with a Dice score of 0.89 , which is 
similar to our study with a Dice score of 0.905 for lumbosacral nerves. Novikov et $\mathrm{al}^{43}$ segmented bones on CT on the basis of the U-Net with a Dice score of 0.95 , which is similar to our study with a Dice score of 0.945 for lumbosacral bones. Furthermore, SPINECT segments lumbosacral nerves and bones in about 13 seconds, which is much shorter than the 30 minutes needed for manual segmentation. In summary, the developed model has the potential to be adopted in the workflow of spinal interventions and minimally invasive spine surgery.

The current study has limitations. First, this pilot study only conducted segmentation on the L5/S1 level because it is one of the most difficult levels for spinal intervention and even for minimally invasive spine surgery. SPINECT will be developed and tested on more levels (eg, L3/L4, L4/L5) and different spinal regions (eg, thoracic, cervical) in the near future. Second, although small vessels surrounding the foramen are critical for tESI, we did not segment pertinent vessels because they were not clear on noncontrast CT. However, semantic segmentation of multiple structures (eg, discs, large vessels, muscles, ligaments) will be integrated into SPINECT. Third, while the subject number and segmentation accuracy are acceptable, more cases may be needed for the accuracy to be further improved. Last, the automatic location of CT planes through deep learning may also help physicians in planning ESIs, and a large observational study exploring its feasibility is also on the way.

\section{CONCLUSIONS}

Deep learning with a 3D U-Net can effectively segment spinal nerves and bones from routine CT. The results of this study suggest that our proposed SPINECT can be used to segment spinal nerves on CT seemingly within near-human expert performance, which is reliable for reconstructing Kambin and safe triangles for tESI.

\section{ACKNOWLEDGMENTS}

We thank our colleagues (Tie Y, Jagadeesan J, Mehrtash A, Zhou H, Kapur T, Kikinis R) in Brigham and Women's Hospital for professional comments and suggestions. We also appreciate the revision contribution by Xiaofei Guan from the Burke Neurologic Institute.

Disclosures: William M. Wells—RELATED: Grant: National Institutes of Health, Comments: P41*; UNRELATED: Employment: Brigham and Women's Hospital, Massachusetts Institute of Technology; Grants/Grants Pending: National Institutes of Health, Comments: P41, R01s.* Shisheng He-RELATED: Grant: Shanghai Shenkang Hospital Development Center.* *Money paid to the institution.

\section{REFERENCES}

1. Mokdad AH, Ballestros K, Echko M, et al; US Burden of Disease Collaborators. The state of US Health, 1990-2016: burden of diseases, injuries, and risk factors among US states. JAMA 2018;319: 1444-72 CrossRef Medline

2. Buchbinder R, van Tulder M, Oberg B, et al; Lancet Low Back Pain Series Working Group. Low back pain: a call for action. Lancet 2018; 391:2384-88 CrossRef Medline

3. Iannuccilli JD, Prince EA, Soares GM. Interventional spine procedures for management of chronic low back pain-a primer. Semin Intervent Radiol 2013;30:307-17 CrossRef Medline

4. ÖKmen K, ÖKmen BM. The efficacy of interlaminar epidural steroid administration in multilevel intervertebral disc disease with chronic low back pain: a randomized, blinded, prospective study. Spine J 2017;17:168-74 CrossRef Medline

5. Cohen SP, Hanling S, Bicket MC, et al. Epidural steroid injections compared with gabapentin for lumbosacral radicular pain: multicenter randomized double blind comparative efficacy study. $B M J$ 2015;350:h1748 CrossRef Medline

6. Lee JH, Shin KH, Bahk SJ, et al. Comparison of clinical efficacy of transforaminal and caudal epidural steroid injection in lumbar and lumbosacral disc herniation: a systematic review and meta-analysis. Spine J 2018;18:2343-53 CrossRef Medline

7. Wei G, Liang J, Chen B, et al. Comparison of transforaminal verse interlaminar epidural steroid injection in low back pain with lumbosacral radicular pain: a meta-analysis of the literature. Int Orthop 2016;40:2533-45 CrossRef Medline

8. Glaser SE, Shah RV. Root cause analysis of paraplegia following transforaminal epidural steroid injections: the 'unsafe' triangle. Pain Physician 2010;13:237-44 Medline

9. Tumialán LM, Madhavan K, Godzik J, et al. The history of and controversy over Kambin's triangle: a historical analysis of the lumbar transforaminal corridor for endoscopic and surgical approaches. World Neurosurg 2019;123:402-08 CrossRef Medline

10. Ra IH, Min WK. Optimal angle of needle insertion for fluoroscopyguided transforaminal epidural injection of L5. Pain Pract 2015;15: 393-99 CrossRef Medline

11. Surange PN. S1 Transforaminal: Technique and Troubleshooting. 2016. https:/www.researchgate.net/publication/303324602_S1_Trans foraminal_Technique_and_Troubleshooting. Accessed January 1, 2016

12. Chen X, Cheng J, Gu X, et al. Development of preoperative planning software for transforaminal endoscopic surgery and the guidance for clinical applications. Int J Comput Assist Radiol Surg 2016;11: 613-20 CrossRef Medline

13. Boswell MV, Trescot AM, Datta S, et al; American Society of Interventional Pain Physicians. Interventional techniques: evidencebased practice guidelines in the management of chronic spinal pain. Pain Physician 2007;10:7-111 Medline

14. Wilkinson I, Cohen SP. Epidural steroids for spinal pain and radiculopathy: a narrative, evidence-based review. Curr Opin Anaesthesiol 2013;26:562-72 CrossRef Medline

15. Kim WJ, Shin HY, Yoo SH, et al. Comparison of epidural spreading patterns and clinical outcomes of transforaminal epidural steroid injection with high-volume injectate via the subpedicular versus the retrodiscal approach. Pain Physician 2018;21:269-78 Medline

16. Park KD, Lee J, Jee $H$, et al. Kambin triangle versus the supraneural approach for the treatment of lumbar radicular pain. Am J Phys Med Rehabil 2012;91:1039-50 CrossRef Medline

17. Fenster AJ, Fernandes K, Brook AL, et al. The safety of CT-guided epidural steroid injections in an older patient cohort. Pain Physician 2016;19:E1139-46 Medline

18. Timpone VM, Hirsch JA, Gilligan CJ, et al. Computed tomography guidance for spinal intervention: basics of technique, pearls, and avoiding pitfalls. Pain Physician 2013;16:369-77 Medline

19. Han Z, Wei B, Leung S, et al. Automated pathogenesis-based diagnosis of lumbar neural foraminal stenosis via deep multiscale multitask learning. Neuroinformatics 2018;16:325-37 CrossRef Medline

20. Pesteie M, Lessoway V, Abolmaesumi P, et al. Automatic localization of the needle target for ultrasound-guided epidural injections. IEEE Trans Med Imaging 2018;37:81-92 CrossRef Medline

21. Yasaka K, Akai H, Abe O, et al. Deep learning with convolutional neural network for differentiation of liver masses at dynamic contrast-enhanced CT: a preliminary study. Radiology 2018;286:887-96 CrossRef Medline

22. Lu JT, Pedemonte S, Bizzo B, et al. DeepSPINE: automated lumbar vertebral segmentation, disc-level designation, and spinal stenosis grading using deep learning. 2018. https://arxiv.org/abs/1807.10215. Accessed July 26, 2018

23. Mazurowski MA, Buda M, Saha A, et al. Deep learning in radiology: an overview of the concepts and a survey of the state of the art. $J$ Magn Reson Imaging 2019;49:939-94 CrossRef Medline 
24. Ronneberger O, Fischer P, Brox T. U-Net: convolutional networks for biomedical image segmentation. 2015. https://arxiv.org/abs/1505.04597. Accessed November 18, 2015

25. Ö Çiçek, Abdulkadir A, Lienkamp SS, et al. 3D U-Net: learning dense volumetric segmentation from sparse annotation. 2016. https://arxiv. org/abs/1606.06650. Accessed October 2, 2016

26. Wang C, Macgillivray T, Macnaught G, et al. A two-stage 3D Unet framework for multi-class segmentation on full resolution image. 2018. https://arxiv.org/pdf/1804.04341.pdf. Accessed April 12, 2018

27. Huang Q, Sun J, Ding H, et al. Robust liver vessel extraction using 3D U-Net with variant dice loss function. Comput Biol Med 2018; 101:153-62 CrossRef Medline

28. Funke J, Tschopp FD, Grisaitis W, et al. Large scale image segmentation with structured loss based deep learning for connectome reconstruction. IEEE Trans Pattern Anal Mach Intel 2018 May 24. [Epub ahead of print] CrossRef Medline

29. Fedorov A, Beichel R, Kalpathy-Cramer J, et al. 3D Slicer as an image computing platform for the Quantitative Imaging Network. Magn Reson Imaging 2012;30:1323-41 CrossRef Medline

30. Zou KH, Warfield SK, Bharatha A, et al. Statistical validation of image segmentation quality based on a spatial overlap index. Acad Radiol 2004;11:178-89 CrossRef Medline

31. He K, Zhang X, Ren S, et al. Delving deep into rectifiers: surpassing human-level performance on ImageNet classification. 2015. https:// arxiv.org/abs/1502.01852. Accessed February 6, 2015

32. Kingma DP, Ba J. Adam: a method for stochastic optimization. Computer Science 2014. https://arxiv.org/abs/1412.6980. Accessed December 22, 2014

33. Cai $Y$, Osman S, Sharma M, et al. Multi-modality vertebra recognition in arbitrary views using 3D deformable hierarchical model. IEEE Trans Med Imaging 2015;34:1676-93 CrossRef Medline

34. Mandell JC, Czuczman GJ, Gaviola GC, et al. The lumbar neural foramen and transforaminal epidural steroid injections: an anatomic review with key safety considerations in planning the percutaneous approach. AJR Am J Roentgenol 2017;209:W1-10 CrossRef Medline

35. Kim C, Choi HE, Kang S. Contrast spreading patterns in retrodiscal transforaminal epidural steroid injection. Ann Rehabil Med 2012; 36:474-79 CrossRef Medline

36. Hardenbrook M, Lombardo S, Wilson MC, et al. The anatomic rationale for transforaminal endoscopic interbody fusion: a cadaveric analysis. Neurosurg Focus 2016;40:E12 CrossRef Medline

37. Guan X, Gu X, Zhang L, et al. Morphometric analysis of the working zone for posterolateral endoscopic lumbar discectomy based on magnetic resonance neurography. J Spinal Disord Tech 2015;28: E78-84 CrossRef Medline

38. Mandell JC, Czuczman GJ, Gaviola GC, et al. The lumbar neural foramen and transforaminal epidural steroid injections: an anatomic review with key safety considerations in planning the percutaneous approach. AJR Am J Roentgenol 2017;209:W26-35 CrossRef Medline

39. Guan X, Fan G, Wu X et al. Diffusion tensor imaging studies of cervical spondylotic myelopathy: a systemic review and meta-analysis. PLoS One 2015;10:e0117707 CrossRef Medline

40. Janssens R, Zeng G, Zheng G. Fully automatic segmentation of lumbar vertebrae from CT images using cascaded 3D fully convolutional networks. 2018. https://www.researchgate.net/publication/325516176_ Fully_automatic_segmentation_of_lumbar_vertebrae_from_CT_images_ using_cascaded_3D_fully_convolutional_networks. Accessed December 5, 2017

41. Dong H, Liu F, Yang G, et al. Automatic brain tumor detection and segmentation using U-Net based fully convolutional networks. 2017. https://www.researchgate.net/publication/318168153_Automatic_ Brain_Tumor_Detection_and_Segmentation_Using_U-Net_Based_ Fully_Convolutional_Networks. Accessed June 22, 2017

42. Feng X, Qing K, Tustison NJ, et al. Deep convolutional neural network for segmentation of thoracic organs-at-risk using cropped 3D images. Med Phys 2019 Mar 4. [Epub ahead of print] CrossRef Medline

43. Novikov AA, Major D, Wimmer M, et al. Deep sequential segmentation of organs in volumetric medical scans. IEEE Trans Med Imaging 2018 Nov 16. [Epub ahead of print] CrossRef Medline 